\section{Antiwindup Design With Guaranteed Regions of Stability: An LMI-Based Approach}

João Manoel Gomes da Silva, Jr. and Sophie Tarbouriech

\begin{abstract}
This note addresses the design of antiwindup gains for obtaining larger regions of stability for linear systems with saturating inputs. Considering that a linear dynamic output feedback has been designed to stabilize the linear system (without saturation), a method is proposed for designing an antiwindup gain that maximizes an estimate of the basin of attraction of the closed-loop system. It is shown that the closed-loop system obtained from the controller plus the antiwindup gain can be modeled by a linear system with a deadzone nonlinearity. A modified sector condition is then used to obtain stability conditions based on quadratic Lyapunov functions. Differently from previous works these conditions are directly in linear matrix inequality form. Some numerical examples illustrate the effectiveness of the proposed design technique when compared with the previous ones.
\end{abstract}

Index Terms-Actuator saturation, antiwindup, linear matrix inequality (LMI), stability.

\section{INTRODUCTION}

The basic idea underlining antiwindup designs for linear systems with saturating actuators is to introduce control modifications in order to recover, as much as possible, the performance induced by a previous design carried out on the basis of the unsaturated system. First results on antiwindup consisted on ad-hoc methods intended to work with standard proportional-integral-derivative (PID) controllers [1], [2] which are commonly used in present commercial controllers. Nonetheless, major improvements in this field have been achieved in the last decade as it can be observed in [3]-[10] among others.

Several results on the antiwindup problem are concerned with achieving global stability properties. Since global results cannot be achieved for open-loop unstable linear systems in the presence of actuator saturation, local results have to be developed. In this context, a key issue is the determination of domains of stability for the closed-loop system (estimates of the basin of attraction). With very few exceptions, most of the local results available in the literature of antiwindup do not provide explicit characterization of the domain of stability.

Recently, in [11] and [12], theoretical results have been developed in order to fill in this gap by providing design algorithms that explicitly optimize a criterion aiming at maximizing a stability domain of the closed-loop system. In [11], the modeling of the nonlinear behavior of the system under saturation is made by using a polytopic differential inclusion and quadratic Lyapunov functions. On the other hand, in [12], based on a transformation of the saturation term in a deadzone nonlinearity, classical sector condition and $S$-procedure techniques are used to derive stability conditions considering both quadratic and Lure type Lyapunov functions. The main drawback of the approaches above is that the conditions allowing to compute the antiwindup gains are given in terms of bilinear matrix inequalities (BMIs). In order to overcome this difficulty, iterative algorithms based on linear matrix inequalities (LMIs) are proposed to solve the synthesis problem. It is well known

Manuscript received June 12, 2003; revised May 19, 2004. Recommended by Associate Editor Y. Ohta. This work was supported by CNPq, Brazil.

J. M. Gomes da Silva, Jr. is with the Department of Electrical Engineering, UFRGS, 90035-190 Porto Alegre-RS, Brazil (e-mail: jmgomes@ eletro.ufrgs.br).

S. Tarbouriech is with LAAS-CNRS, 31077 Toulouse cedex 4, France (e-mail: tarbour@laas.fr).

Digital Object Identifier 10.1109/TAC.2004.841128 that, in general this kind of approach leads only to local optimal solutions and are very sensitive to the initialization [13].

In this note, we propose a new approach for synthesizing antiwindup gains taking into account an associated region of asymptotic stability for the closed-loop system. As pointed in [11] and [12], this problem is implicitly related to the problem of enlarging the region of attraction by means of antiwindup schemes. Our results are based on the proposition of a modified sector condition. Based on this new sector condition, the stability conditions can be formulated directly in LMI form. It is shown that the results of [12] appears as a particular case of the new conditions. On the other hand, the results are potentially less conservative than the ones of [11].

The note is organized as follows. The problem to be treated is formally stated in Section II. The theoretical results allowing to determine the antiwindup gain and an associated region of stability are presented in Section III. Section IV is devoted to the presentation of a convex optimization problem in order to compute the antiwindup gain aiming at enlarging the basin of attraction of the closed-loop system. The extension of the methodology to the case where the inputs are delayed is presented in Section V. The effectiveness of the proposed approach is illustrated by means of examples in Section VI. Some concluding remarks ends this note.

Notations: The $i$ th component of a vector $x$ is denoted by $x_{(i)}$. The elements of a matrix $A \in \Re^{m \times n}$ are denoted by $A_{(i, l)}, i=1, \ldots, m$, $l=1, \ldots, n . A_{(i)}$ denotes the $i$ th row of matrix $A$. For two symmetric matrices, $A$ and $B, A>B$ means that $A-B$ is positive definite. $A^{\prime}$ denotes the transpose of $A$. $\operatorname{diag}(x)$ denotes a diagonal matrix obtained from vector $x . I_{m}$ denotes the $m$-order identity matrix. $C o\{$.$\} denotes$ a convex hull.

\section{PROBLEM STATEMENT}

Consider the continuous-time linear system

$$
\left\{\begin{array}{l}
\dot{x}(t)=A x(t)+B u(t) \\
y(t)=C x(t)
\end{array}\right.
$$

where $x(t) \in \Re^{n}, u(t) \in \Re^{m}, y(t) \in \Re^{p}$ are the state, the input, and the measured output vectors, respectively. Matrices $A, B$ and $C$ are real constant matrices of appropriate dimensions. Pairs $(A, B)$ and $(C, A)$ are assumed to be controllable and observable, respectively.

Considering system (1), we assume that an $n_{c}$-order dynamic output stabilizing compensator

$$
\begin{aligned}
\dot{\eta}(t) & =A_{c} \eta(t)+B_{c} y(t) \\
v_{c}(t) & =C_{c} \eta(t)+D_{c} y(t)
\end{aligned}
$$

where $\eta(t) \in \Re^{n} c$ is the controller state, $u_{c}(t)=y(t)$ is the controller input, and $v_{c}(t)$ is the controller output, has been designed in order to guarantee some performance requirements and the stability of the closed-loop system in the absence of control saturation.

Suppose now that the input vector $u$ is subject to amplitude limitations as follows:

$$
-u_{0(i)} \leq u_{(i)} \leq u_{0(i)}, \quad u_{0(i)}>0, \quad i=1, \ldots, m .
$$

In consequence of the control bounds, the actual control signal to be injected in the system is a saturated one, that is

$$
u(t)=\operatorname{sat}\left(v_{c}(t)\right)=\operatorname{sat}\left(C_{c} \eta(t)+D_{c} C x(t)\right)
$$

where each component of $\operatorname{sat}\left(v_{c}(t)\right)$ is defined $\forall i=1, \ldots, m$ by

$$
\operatorname{sat}\left(v_{c(i)}(t)\right) \triangleq \operatorname{sign}\left(v_{c(i)}(t)\right) \min \left(\left|v_{c(i)}(t)\right|, u_{0(i)}\right) .
$$


In order to mitigate the undesirable effects of windup caused by input saturation, an antiwindup term $E_{c}\left(\operatorname{sat}\left(v_{c}(t)\right)-v_{c}(t)\right)$ can be added to the controller [5]. Thus, considering the dynamic controller and this antiwindup strategy, the closed-loop system reads

$$
\begin{aligned}
\dot{x}(t) & =A x(t)+B \operatorname{sat}\left(v_{c}(t)\right) \\
y(t) & =C x(t) \\
\dot{\eta}(t) & =A_{c} \eta(t)+B_{c} y(t)+E_{c}\left(\operatorname{sat}\left(v_{c}(t)\right)-v_{c}(t)\right) \\
v_{c}(t) & =C_{c} \eta(t)+D_{c} y(t) .
\end{aligned}
$$

Define now an extended state vector $\xi(t)=\left[x(t)^{\prime} \eta(t)^{\prime}\right]^{\prime} \in \Re^{n+n_{c}}$ and the following matrices:

$$
\begin{aligned}
& \mathbb{A}=\left[\begin{array}{cc}
A+B D_{c} C & B C_{c} \\
B_{c} C & A_{c}
\end{array}\right] \quad \mathbb{B}=\left[\begin{array}{l}
B \\
0
\end{array}\right] \\
& \mathbb{R}=\left[\begin{array}{c}
0 \\
I_{n_{c}}
\end{array}\right] \quad \mathbb{K}=\left[\begin{array}{ll}
D_{c} C & C_{c}
\end{array}\right] .
\end{aligned}
$$

Hence, from the previous definitions, the closed-loop system reads

$$
\dot{\xi}(t)=A \xi(t)-\left(\mathbb{B}+\mathbb{R} E_{c}\right) \psi(\mathbb{K} \xi(t))
$$

with the function $\psi(v) \triangleq v-s a t(v)$. Note that, in this case, $\psi(v)$ corresponds to a decentralized deadzone nonlinearity $\psi(v)=\left[\psi\left(v_{(1)}\right) \ldots \psi\left(v_{(m)}\right)\right]^{\prime}$, where $\forall i=1, \ldots, m$

$$
\psi\left(v_{(i)}\right) \triangleq \begin{cases}v_{(i)}-u_{0(i)} & \text { if } v_{(i)}>u_{0(i)} \\ 0 & \text { if }-u_{0(i)} \leq v_{(i)} \leq u_{0(i)} . \\ v_{(i)}+u_{0(i)} & \text { if } v_{(i)}<-u_{0(i)}\end{cases}
$$

Matrix A is supposed to be Hurwitz, i.e., in the absence of control bounds, the closed-loop system would be globally stable.

The problem we aim to solve throughout this note is then summarized as follows.

Problem 1: Determine the antiwindup gain matrix $E_{c}$ and a region of asymptotic stability, as large as possible, for the closed-loop system (7).

Of course, the implicit objective in Problem 1 is to optimize the size of the basin of attraction for the closed-loop system (7) over the choice of the gain matrix $E_{c}$. This can be accomplished indirectly by searching for an antiwindup gain $E_{c}$ that leads to a region of stability for the closed-loop system as large as possible.

\section{STABILITY CONDITIONS}

Consider a matrix $G \in \Re^{m \times\left(n+n_{c}\right)}$ and define the following polyhedral set:

$$
\mathcal{S} \triangleq\left\{\xi \in \Re^{n+n_{c}} ;\left|\left(\mathbb{K}_{(i)}-G_{(i)}\right) \xi\right| \leq u_{0(i)}, i=1, \ldots, m\right\} .
$$

Regarding the nonlinearity (8) and the set $\mathcal{S}$, the following Lemma can be stated.

Lemma 1: Consider the function $\psi(v)$ defined in (8). If $\xi \in \mathcal{S}$ then the relation

$$
\psi(\mathbb{K} \xi)^{\prime} T[\psi(\mathbb{K} \xi)-G \xi] \leq 0
$$

is verified for any matrix $T \in \Re^{m \times m}$ diagonal and positive definite.

Proof: Consider the three cases that follow.

a) $-u_{0(i)} \leq \mathbb{K}_{(i)} \xi \leq u_{0(i)}$.

In this case, by definition, $\psi\left(\mathbb{K}_{(i)} \xi\right)=0$ and then $\psi\left(\mathbb{K}_{(i)} \xi\right) T_{(i, i)}\left[\psi\left(\mathbb{K}_{(i)} \xi\right)-G_{(i)} \xi\right]=0$.

b) $\mathbb{K}_{(i)} \xi>u_{0(i)}$. In this case, $\psi\left(\mathbb{K}_{(i)} \xi\right)=\mathbb{K}_{(i)} \xi-u_{0(i)}$. If $\xi \in \mathcal{S}$, it follows that $\mathbb{K}_{(i)} \xi-G_{(i)} \xi \leq u_{0(i)}$. Hence, it follows that $\psi\left(\mathbb{K}_{(i)} \xi\right)-G_{(i)} \xi=\mathbb{K}_{(i)} \xi-u_{0(i)}-G_{(i)} \xi \leq 0$ and, since in this case $\psi\left(\mathbb{K}_{(i)} \xi\right)>0$, one gets $\psi\left(\mathbb{K}_{(i)} \xi\right) T_{(i, i)}\left[\psi\left(\mathbb{K}_{(i)} \xi\right)-\right.$ $\left.G_{(i)} \xi\right] \leq 0, \forall T_{(i, i)}>0$.

c) $\mathbb{K}_{(i)} \xi<-u_{0(i)}$. In this case, $\psi\left(\mathbb{K}_{(i)} \xi\right)=\mathbb{K}_{(i)} \xi+u_{0(i)}$. If $\xi \in \mathcal{S}$, it follows that $\mathbb{K}_{(i)} \xi-G_{(i)} \xi \geq-u_{0(i)}$. Hence, it follows that $\psi\left(\mathbb{K}_{(i)} \xi\right)-G_{(i)} \xi=\mathbb{K}_{(i)} \xi+u_{0(i)}-G_{(i)} \xi \geq 0$ and, since in this case $\psi\left(\mathbb{K}_{(i)} \xi\right)<0$, one gets $\psi\left(\mathbb{K}_{(i)} \xi\right) T_{(i, i)}\left[\psi\left(\mathbb{K}_{(i)} \xi\right)-\right.$ $\left.G_{(i)} \xi\right] \leq 0, \forall T_{(i, i)}>0$.

From these three cases, provided that $\xi \in \mathcal{S}$, we can conclude that $\psi\left(\mathbb{K}_{(i)} \xi\right) T_{(i, i)}\left[\psi\left(\mathbb{K}_{(i)} \xi\right)-G_{(i)} \xi\right] \leq 0, \forall T_{(i, i)}>0, \forall i=1, \ldots, m$, from where follows (10).

Consider now, as a Lyapunov candidate function, the quadratic function

$$
V(\xi(t))=\xi(t)^{\prime} P \xi(t), \quad P=P^{\prime}>0, \quad P \in \Re^{\left(n+n_{c}\right) \times\left(n+n_{c}\right)} .
$$

Theorem 1: If there exist a symmetric positive-definite matrix $W \in$ $\Re^{\left(n+n_{c}\right) \times\left(n+n_{c}\right)}$, a matrix $Y \in \Re^{m \times\left(n+n_{c}\right)}$, a matrix $Z \in \Re^{n_{c} \times m}$ and a diagonal positive-definite matrix $S \in \Re^{m \times m}$ satisfying

$$
\begin{aligned}
& {\left[\begin{array}{cc}
W \mathbb{A}^{\prime}+\mathrm{A} W & \mathbb{B} S+\mathbb{R} Z-Y^{\prime} \\
S \mathbb{B}^{\prime}+Z^{\prime} \mathbb{R}^{\prime}-Y & -2 S
\end{array}\right]<0} \\
& {\left[\begin{array}{cc}
W & W \mathbb{K}_{(i)}^{\prime}-Y_{(i)}^{\prime} \\
\mathbb{K}_{(i)} W-Y_{(i)} & u_{0(i)}^{2}
\end{array}\right] \geq 0, \quad i=1, \ldots, m}
\end{aligned}
$$

then the gain matrix $E_{c}=Z S^{-1}$ is such that the ellipsoid $\mathcal{E}(P)=$ $\left\{\xi \in \Re^{n+n_{c}} ; \xi^{\prime} P \xi \leq 1\right\}$, with $P=W^{-1}$, is an asymptotic stability region for system (6).

Proof: The satisfaction of relations (13) implies that the set $\mathcal{E}(P)$ is included in the polyhedral set $\mathcal{S}$ defined as in (9) with $G=Y P$ [13], [14]. Hence, from Lemma 1 , for all $\xi(t) \in \mathcal{E}(P)$ it follows that $\psi(\mathbb{K} \xi(t))=\mathbb{K} \xi(t)-\operatorname{sat}(\mathbb{K} \xi(t))$ satisfies the sector condition (10). By considering the quadratic candidate Lyapunov function as defined in (11) and by computing its time-derivative along the trajectories of system (7) one gets

$$
\dot{V}(\xi(t))=\xi(t)^{\prime}\left(\mathbb{A}^{\prime} P+P A\right) \xi(t)-2 \xi(t)^{\prime} P\left(\mathbb{B}+\mathbb{R} E_{c}\right) \psi(\mathbb{K} \xi(t)) .
$$

Thus, by using the sector condition (10), it follows that

$$
\begin{aligned}
\dot{V}(\xi(t)) \leq \dot{V}(\xi(t))-2 \psi(\mathbb{K} \xi(t))^{\prime} T \psi & (\mathbb{K} \xi(t)) \\
& +2 \psi(\mathbb{K} \xi(t))^{\prime} T G \xi(t)
\end{aligned}
$$

for any $\xi \in \mathcal{E}(P)$.

The right-hand side of (14) can be written in the form

$$
\left[\begin{array}{ll}
\xi(t)^{\prime} & -\psi(\mathbb{K} \xi(t))^{\prime}
\end{array}\right]\left[\begin{array}{ll}
M_{1} & M_{2} \\
M_{2}^{\prime} & M_{3}
\end{array}\right]\left[\begin{array}{c}
\xi(t) \\
-\psi(\mathbb{K} \xi(t))
\end{array}\right]
$$

where

$$
\begin{aligned}
& M_{1}=\mathbb{A}^{\prime} P+P A \\
& M_{2}=P \mathbb{B}+P \mathbb{R} E_{c}-G^{\prime} T . \\
& M_{3}=-2 T
\end{aligned}
$$

Note now that pre- and postmultiplying (12) by

$$
\left[\begin{array}{ll}
P & 0 \\
0 & T
\end{array}\right]
$$

and considering $W=P^{-1}, S=T^{-1}$, and $Z=E_{c} T^{-1}$, it follows that

$$
\left[\begin{array}{ll}
M_{1} & M_{2} \\
M_{2}^{\prime} & M_{3}
\end{array}\right]<0
$$

Hence, if (12) is satisfied one has $\dot{V}(\xi(t))<0$. Since this reasoning is valid $\forall \xi(t) \in \mathcal{E}(P), \xi(t) \neq 0$, it follows that $\mathcal{E}(P)$ is a positively 
invariant and contractive region for system (7) which means that for any $\xi(0) \in \mathcal{E}(P)$, the corresponding trajectory converges asymptotically to the origin, i.e., $\mathcal{E}(P)$ is a region of asymptotic stability for the closedloop system (7).

Theorem 1 gives a condition for the local stability of system (6) inside an ellipsoidal region in the state-space. On the other hand, the following corollary, concerning a global stability condition, can be stated.

Corollary 1: If there exist a symmetric positive-definite matrix $W \in \Re^{\left(n+n_{c}\right) \times\left(n+n_{c}\right)}$, a diagonal positive-definite matrix $S \in \Re^{m \times m}$ and a matrix $Z \in \Re^{n_{c} \times m}$ satisfying

$$
\left[\begin{array}{cc}
W A^{\prime}+A W & \mathbb{B} S+\mathbb{R} Z-W \mathbb{K}^{\prime} \\
S \mathbb{B}^{\prime}+Z^{\prime} \mathbb{R}^{\prime}-\mathbb{K} W & -2 S
\end{array}\right]<0
$$

then, for $E_{c}=Z S^{-1}$, system (7) is globally asymptotically stable.

Proof: Consider $G=\mathbb{K}$. It follows that (10) is verified for all $\xi \in$ $\Re^{n+n_{c}}$. In this case, (15) corresponds to (12) and the global asymptotic stability follows.

Remark 1: The result in [12] appears as a particular case of Theorem 1. In that paper, $\psi(\mathbb{K} \xi)$ satisfies the "classical" sector condition: $\psi(\mathbb{K} \xi)^{\prime} T[\psi(\mathbb{K} \xi)-\Lambda \mathbb{K} \xi] \leq 0, \forall \xi \in S\left(\mathbb{K}, u_{0}^{\lambda}\right)$, where $\Lambda$ is a positive diagonal matrix and the set $S\left(\mathbb{K}, u_{0}^{\lambda}\right)$ is a polyhedral set defined as follows:

$$
S\left(\mathbb{K}, u_{0}^{\lambda}\right)=\left\{\xi \in \Re^{n+n_{c}} ;\left|\mathbb{K}_{(i)} \xi\right| \leq \frac{u_{0(i)}}{1-\Lambda_{(i, i)}}, i=1, \ldots, m\right\} .
$$

Following a similar procedure to the one applied in the proof of Theorem 1, the following conditions are obtained:

$$
\begin{aligned}
& {\left[\begin{array}{cc}
W \mathbb{A}^{\prime}+A W & \mathbb{B} S+\mathbb{R} Z-W \mathbb{K}^{\prime} \Lambda \\
S \mathbb{B}^{\prime}+Z^{\prime} \mathbb{R}^{\prime}-\Lambda \mathbb{K} W & -2 S
\end{array}\right]<0} \\
& {\left[\begin{array}{cc}
W & \left(1-\Lambda_{(i, i)}\right) W \mathbb{K}_{(i)}^{\prime} \\
\left(1-\Lambda_{(i, i)}\right) \mathbb{K}_{(i)} W & \gamma u_{0(i)}^{2}
\end{array}\right] \geq 0,}
\end{aligned}
$$

Note that these matrix inequalities are bilinear in variables $W$ and $\Lambda$. It is easy to see that (17) and (18) corresponds to the conditions of Theorem 1 by taking $G=\Lambda \mathbb{K}$. Hence, all the solutions obtained considering (17) and (18) are also feasible solutions for (12) and (13).

Remark 2: Regarding the approach developed in [11], we can state the following comments.

- The stabilization condition proposed in [11] appears as the verification of a set of BMIs in the problem variables. Considering $m$-inputs, $2^{m}+m$ BMIs should be verified. In this case, the search for the antiwindup gain that maximizes the region of attraction should be done by means of iterative schemes, based on the solution of LMI problems. It is well-known that this corresponds to a standard ad-hoc solution when conditions are formulated in BMI form. In this case, the method is very sensitive to the initial considered guess and only local sub-optimality can be ensured. In this note, the stability condition appears directly in LMI form. Considering $m$-inputs, only $m+1$ LMIs should be verified. Hence, the problem solution can be obtained by solving a simple convex LMI problem. Furthermore, in this case the solution is optimal, no initial guesses neither iterative schemes are needed.

- The polyhedral sets $\mathcal{L}(H)$ (in [11]) and $\mathcal{S}$ [defined in (9)] play the same role in the sense that they represent a set where the ellipsoidal contractive set $\mathcal{E}(P)$ must be included in order to ensure that the representation of the saturated system [the polytopic linear differential inclusion (LDI) approximation in [11] or the proposed modified sector in the current note] is valid.

- Condition of global asymptotic stability (in the case where the open-loop system is stable) is directly obtained as shown in
Corollary 1 , which is not possible by using a polytopic model as in [11].

Remark 3: Considering the single input case, $T=t>0$ is a scalar and condition (12) can be rewritten as

$$
\begin{aligned}
(\tilde{\mathrm{A}}+\tilde{\mathbb{B}}(\mathbb{K}-G))^{\prime} P+P(\tilde{\mathbb{A}}+\tilde{\mathbb{B}}(\mathbb{K}-G))+ \\
0.5 t^{-1}\left(P \tilde{\mathbb{B}}+G^{\prime} t\right)\left(P \tilde{\mathbb{B}}+G^{\prime} t\right)^{\prime}<0
\end{aligned}
$$

where $\tilde{\mathbb{B}}=\mathbb{B}+\mathbb{R} E_{c}$ and $\tilde{\mathbb{A}}=\mathrm{A}_{0}-\mathbb{R} E_{c} \mathbb{K}$, with $A_{0}=\left[\begin{array}{cc}A & 0 \\ B_{c} C & A_{c}\end{array}\right]$.

Defining $H=(\mathbb{K}-G)$ it follows that the satisfaction of conditions (12) and (13) imply the satisfaction of the necessary and sufficient ellipsoidal invariance condition for single input linear systems subject to actuator saturation, as stated in [15]. Note that in this case, condition (12) implies that $(\tilde{A}+\tilde{\mathbb{B}} H)^{\prime} P+P(\tilde{A}+\tilde{B} H)<0$ and condition (13) is equivalent to $\mathcal{E}(P) \subset \mathcal{L}(H)$.

\section{ANTIWINDUP GAIN COMPUTATION}

Based on the result stated in Theorem 1, in this section we aim to present a convex optimization problem in order to obtain an antiwindup gain matrix that ensures the local stability of the closed-loop system in a region of the state space $\Re^{n+n_{c}}$. In this case, we are interested in one of the following cases.

1) A set of admissible initial conditions, $\Xi_{0} \subset \Re^{n+n_{c}}$, for which asymptotic stability must be ensured, is given.

2) We aim to design the antiwindup gain in order to maximize an estimate of the basin of attraction associated to it. In other words, we want to compute $E_{c}$ such that the associated region of asymptotic stability is as large as possible considering some size criterion.

Both cases can be addressed if we consider a set $\Xi_{0}$ with a given shape and a scaling factor $\beta$. For example, let $\Xi_{0}$ be defined as a polyhedral set described by the convex hull of its vertices

$$
\Xi_{0} \triangleq \operatorname{Co}\left\{v_{1}, v_{2}, \ldots, v_{n_{r}}\right\}, \quad v_{r} \in \Re^{n+n_{c}}, \quad r=1, \ldots, n_{r} .
$$

Recalling Theorem 1, we aim at searching for matrices $W, Y, S, Z$ in order to obtain $\beta \Xi_{0} \subset \mathcal{E}(P)$. In case 1), this problem reduces to a feasibility problem with $\beta=1$ whereas in case 2 ), the goal will be to maximize $\beta$. Note that in the last case, $\Xi_{0}$ defines the directions in which we want to maximize $\mathcal{E}(P)$. The problem of maximizing $\beta$ can be accomplished by solving the following convex optimization problem (eigenvalue problem [14]):

$$
\left\{\begin{array}{c}
\min _{W, Z, S, Y, \mu} \mu \\
\text { subject to } \\
(i)\left[\begin{array}{cc}
\mu & v_{r}^{\prime} \\
v_{r} & W
\end{array}\right] \geq 0, \quad r=1, \ldots, n_{r} \\
\text { LMIs (12) and (13). }
\end{array}\right.
$$

Considering $\beta=1 / \sqrt{\mu}$, the minimization of $\mu$ implies the maximization of $\beta$. The satisfaction of the inclusion relation $\beta \Xi_{0} \subset \mathcal{E}(P)$ is ensured by the LMI $(i)$. It should be noticed that other criteria associated to the size of the set $\mathcal{E}(P)$, (e.g. the volume or the size of the minor axis) can be adopted in order to maximize the stability region.

A constraint of antiwindup gain limitation can be added to the optimization problem (19) as follows. Since $E_{c}=Z S^{-1}$, it follows that $E_{c(i, j)}=Z_{(i, j)} S_{(j, j)}^{-1}$. Hence, if $\left[\begin{array}{cc}S_{(j, j)} \sigma & Z_{(i, j)} \\ Z_{(i, j)} & S_{(j, j)}\end{array}\right] \geq 0$ by the Schur's complement one has $\sigma-Z_{(i, j)} S_{(j, j)}^{-1} Z_{(i, j)} S_{(j, j)}^{-1} \geq 0$ which 
ensures that $\left(E_{c(i, j)}\right)^{2} \leq \sigma$. By the same reasoning, structural constraints on $E_{c}$ can be taken into account in (19) by fixing some of the elements of matrix $Z_{(i, j)}$ as zero.

\section{EXTENSION TO THE INPUT-DELAYED CASE}

We consider now the case where the input is delayed by $\tau$, i.e., the open-loop system reads

$$
\left\{\begin{array}{l}
\dot{x}(t)=A x(t)+B u(t-\tau) \\
y(t)=C x(t)
\end{array} .\right.
$$

In this case, given a dynamic controller as in (2), the closed-loop system in the presence of input saturation can be rewritten as

$$
\dot{\xi}(t)=\mathrm{A}_{0} \xi(t)+\mathrm{A}_{d} \xi(t-\tau)-\mathbb{B} \psi(\mathbb{K} \xi(t-\tau))-\mathbb{R} E_{c} \psi(\mathbb{K} \xi(t))
$$

where $A_{0}=\left[\begin{array}{cc}A & 0 \\ B_{c} C & A_{c}\end{array}\right], \mathbb{A}_{d}=\left[\begin{array}{cc}B D_{c} C & B C_{c} \\ 0 & 0\end{array}\right]$ and $\xi(t), \mathbb{B}, \mathbb{K}$, and $\mathbb{R}$ are defined as in Section II. System (21) admits an augmented initial condition $\xi\left(t_{0}+\theta\right)=\phi_{\xi}(\theta), \forall \theta \in[-\tau, 0]$. As in Section II, matrix $A=A_{0}+A_{d}$ is supposed Hurwitz by construction.

Problem 1 considering (21) has been recently addressed in [16]. In that paper, the stabilization conditions were stated by using a "classical" sector condition, as detailed in Remark 1. As a consequence, these conditions are BMIs. The solution of the problem is then carried out by relaxation schemes.

Following now a similar development to the one used in [16], but using the result stated in Lemma 1, the following Theorem can be stated.

Theorem 1: If there exist symmetric positive definite matrices $W$, $X, R, H, U \in \Re^{\left(n+n_{c}\right) \times\left(n+n_{c}\right)}$ and $D \in \Re^{m \times m}$, a matrix $Y \in$ $\Re^{m \times\left(n+n_{c}\right)}$, a matrix $Z \in \Re^{n_{c} \times m}$, and a diagonal positive-definite matrix $S \in \Re^{m \times m}$ satisfying inequality (13) and

$$
\left[\begin{array}{ccc}
Q_{1} & Q_{2} & Q_{3} \\
\star & Q_{4} & 0 \\
\star & \star & Q_{5}
\end{array}\right]<0
$$

where

$$
Q_{1}=\left[\begin{array}{ccc}
\Gamma & \star & \star \\
Y-Z^{\prime} \mathbb{R}^{\prime} & -2 S+D & \star \\
-S \mathbb{B}^{\prime} & 0 & -D
\end{array}\right]<0
$$

$\Gamma=W\left(\mathrm{~A}_{0}+\mathrm{A}_{d}\right)^{\prime}+\left(\mathrm{A}_{0}+\mathrm{A}_{d}\right) W+\tau \mathrm{A}_{d}(X+R+H+U) \mathbb{A}_{d}^{\prime}$

$$
\begin{aligned}
Q_{2} & =\left[\begin{array}{cc}
\tau W \mathbb{A}_{0}^{\prime} & \tau W \mathbb{A}_{d}^{\prime} \\
0 & 0 \\
0 & 0
\end{array}\right] \\
Q_{3} & =\left[\begin{array}{cc}
0 & 0 \\
\tau S \mathbb{B}^{\prime} & \tau Z^{\prime} \\
0 & 0
\end{array}\right] \\
Q_{4} & =\left[\begin{array}{cc}
-\tau X & 0 \\
0 & -\tau R
\end{array}\right] \\
Q_{5} & =\left[\begin{array}{cc}
-\tau H & 0 \\
0 & -\tau U
\end{array}\right]
\end{aligned}
$$

$G=Y W^{-1}$, then the gain matrix $E_{c}=Z S^{-1}$ ensures that for any initial condition $\phi_{\xi}(\theta)$ belonging to the set $\Phi_{0}=\left\{\phi \in \mathcal{C}_{2 \tau}^{v} ;\|\phi\|_{c}^{2} \leq \delta\right\}$, with $\delta^{-1}=\lambda_{\max }\left(W^{-1}\right)+$ $\tau \lambda_{\max }\left(S^{-1} D S^{-1}\right)\|G\|^{2}+\left(3 \tau^{2} / 2\right)\left[\lambda_{\max }\left(\mathrm{A}_{d}^{\prime} R^{-1} \mathrm{~A}_{d}\right) \quad+\right.$ $\left.\lambda_{\max }\left(\mathbb{B}^{\prime} H^{-1} \mathbb{B}\right)\|G\|^{2}\right]+\left(\tau^{2} / 2\right)\left[\lambda_{\max }\left(\mathbb{A}_{0}^{\prime} X^{-1} \mathbb{A}_{0}\right)+\lambda_{\max }\left(E_{c}^{\prime} \mathbb{R}^{\prime}\right.\right.$ $\left.\left.U^{-1} \mathbb{R} E_{c}\right)\|G\|^{2}\right]$, the trajectories of the closed-loop system converge asymptotically to the origin.

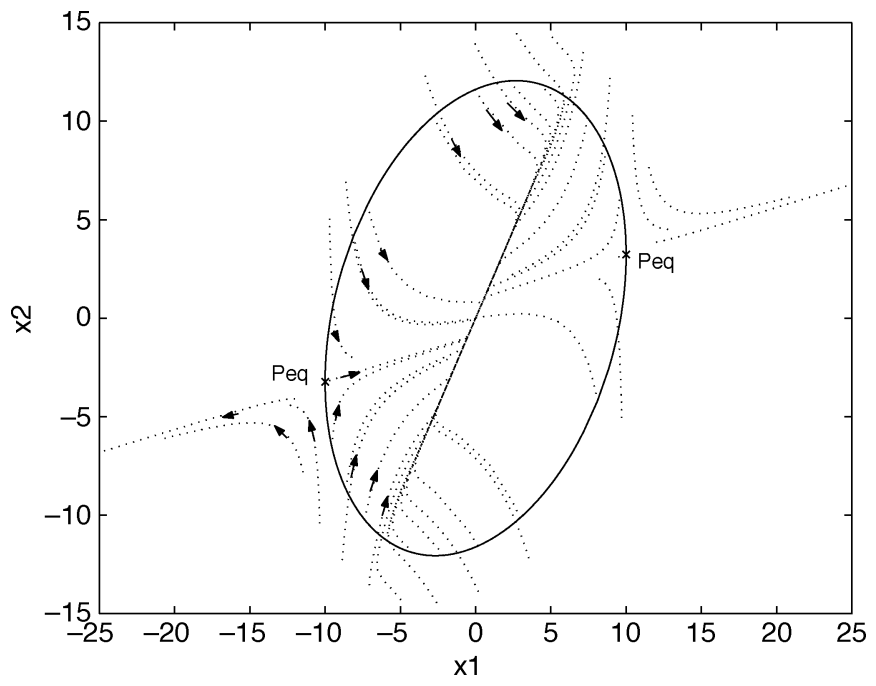

Fig. 1. Stability domain and rajectories for $E_{c}=0.1269$.

The proof of the Theorem 1 is omitted for reasons of place. It can be obtained from the application of Lemma 1 to the proof of the results in [16], as detailed in [17].

Note that, differently from [16], the condition (22) and the inclusion relation (13) are now LMIs in variables $W, X, R, H, U, D, S, Y$, and $Z$. Furthermore, (22) is simpler than the equivalent one in [16]. The synthesis of $E_{c}$ aiming at the maximization of the set of admissible initial conditions can then be carried out by using the optimization criterion discussed in [16].

\section{ILLUSTRATIVE EXAMPLES}

Example 1: Consider the following linear open-loop unstable system [12]:

$$
\begin{aligned}
& \dot{x}(t)=0.1 x(t)+u(t) \\
& y(t)=x(t)
\end{aligned}
$$

and the stabilizing PI controller

$$
\begin{aligned}
& \dot{\eta}_{c}(t)=-0.2 y(t) \\
& v_{c}(t)=\eta_{c}(t)-2 y(t) .
\end{aligned}
$$

Let the shape set $\Xi_{0}$ be defined by a square region in the space $\Re^{2}$

$$
\Xi_{0}=C o\left\{\left[\begin{array}{l}
1 \\
1
\end{array}\right] ;\left[\begin{array}{c}
1 \\
-1
\end{array}\right] ;\left[\begin{array}{c}
-1 \\
1
\end{array}\right] ;\left[\begin{array}{l}
-1 \\
-1
\end{array}\right]\right\} \text {. }
$$

Considering the control bound $u_{0}=1$ and a scaling factor $\beta$, we aim to compute an antiwindup gain $E_{c}$ in order to obtain a region of stability $\beta \Xi_{0} \subset \mathcal{E}(P)$ with $\beta$ as large as possible. Hence, solving the LMI problem (19) one obtains

$$
\beta=6.5956 \quad W=\left[\begin{array}{ll}
99.9997 & 32.3392 \\
32.3392 & 145.3100
\end{array}\right] \quad E_{c}=0.1269 .
$$

It is worth noticing that, with the computed $E_{c}$ gain, the closed-loop system presents unstable equilibrium points in $\pm\left[\begin{array}{ll}10 & 3.2339\end{array}\right]^{\prime}$. These points are very close to the boundary of the stability domain obtained, thus showing that the method can provide a potentially good approximation of the basin of attraction. Fig. 1 depicts the stability domain obtained and state closed-loop trajectories. As expected, the value of $\beta$ obtained is bigger than the one obtained in [12] from the classical sector condition $(\beta=5.6872)$. On the other hand, considering $E_{c}=0$ [i.e., considering $Z=0$ in (12)] one obtains $\beta=4.3514$, which shows that 


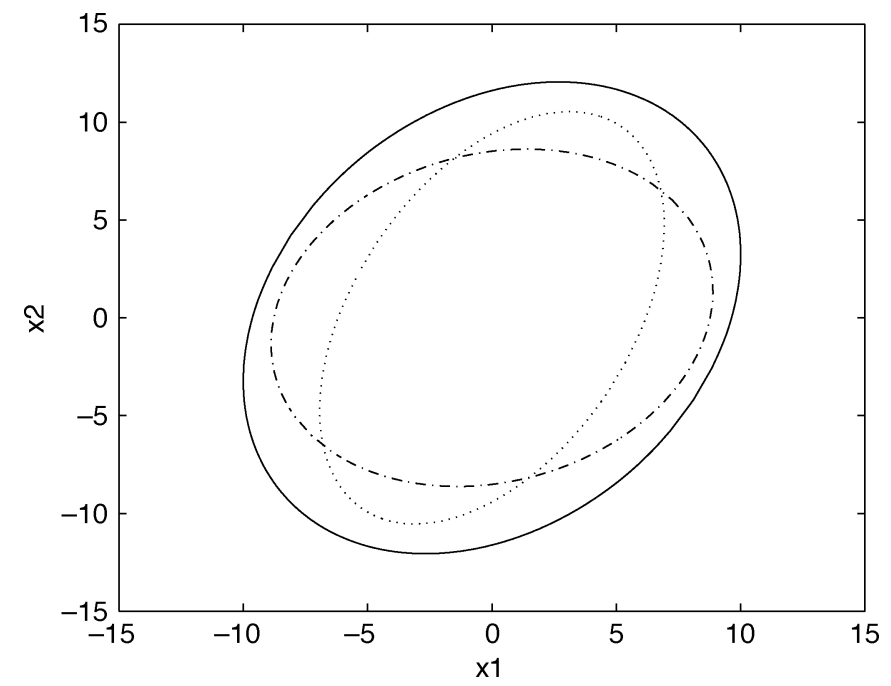

Fig. 2. Proposed approach (continuous). Approach presented in [12] (dashed-dotted). $E_{c}=0$ (dotted).

the antiwindup gain is useful for enlarging the basin of attraction of the closed-loop system. Fig. 2, depicts the domains of stability obtained in the previous cases.

Example 2: Consider the following multivariable linear open-loop unstable system described by the following matrices [12]:

$$
A=\left[\begin{array}{ll}
0.1 & -0.1 \\
0.1 & -3
\end{array}\right] \quad B=\left[\begin{array}{ll}
5 & 0 \\
0 & 1
\end{array}\right] \quad C=I_{2}
$$

and the stabilizing dynamic controller given by

$$
\begin{array}{ll}
A_{c}=\left[\begin{array}{ll}
-171.2 & 27.2 \\
-68 & -626.8
\end{array}\right] \quad B_{c}=\left[\begin{array}{ll}
-598.2 & 5.539 \\
-4.567 & 149.8
\end{array}\right] \\
C_{c}=\left[\begin{array}{ll}
0.146 & 0.088 \\
-6.821 & -5.67
\end{array}\right] \quad D_{c}=0_{2} .
\end{array}
$$

The control bounds are given by $u_{0}=\left[\begin{array}{ll}5 & 2\end{array}\right]^{\prime}$ and we consider

$$
\Xi_{0}=C o\left\{\left[\begin{array}{l}
1 \\
1 \\
0 \\
0
\end{array}\right]\left[\begin{array}{c}
1 \\
-1 \\
0 \\
0
\end{array}\right]\left[\begin{array}{c}
-1 \\
1 \\
0 \\
0
\end{array}\right]\left[\begin{array}{c}
-1 \\
-1 \\
0 \\
0
\end{array}\right]\right\}
$$

This choice corresponds to maximize the estimate of the region of attraction in the states of the plant. The solution of (19) gives the following optimal results:

$$
\beta=250.8677 \quad E_{c}=10^{4}\left[\begin{array}{rr}
2.4176 & -0.0169 \\
0.3590 & 0.0011
\end{array}\right] .
$$

As in the previous example, the value of $\beta$ obtained is bigger than the one obtained in [12] from the classical sector condition ( $\beta=124.71$ ). Fig. 3 depicts the projection of the stability domains on the plane $\left(x_{1}, x_{2}\right)$ (corresponding to the states of the plant) obtained from each approach. With the computed $E_{c}$ gain, the closed-loop system presents unstable equilibrium points in $\pm\left[\begin{array}{llll}259.3103 & 9.3103 & -70.3493 & 21.3140\end{array}\right]^{\prime}$. These points are also very close to the boundary of the stability domain obtained.

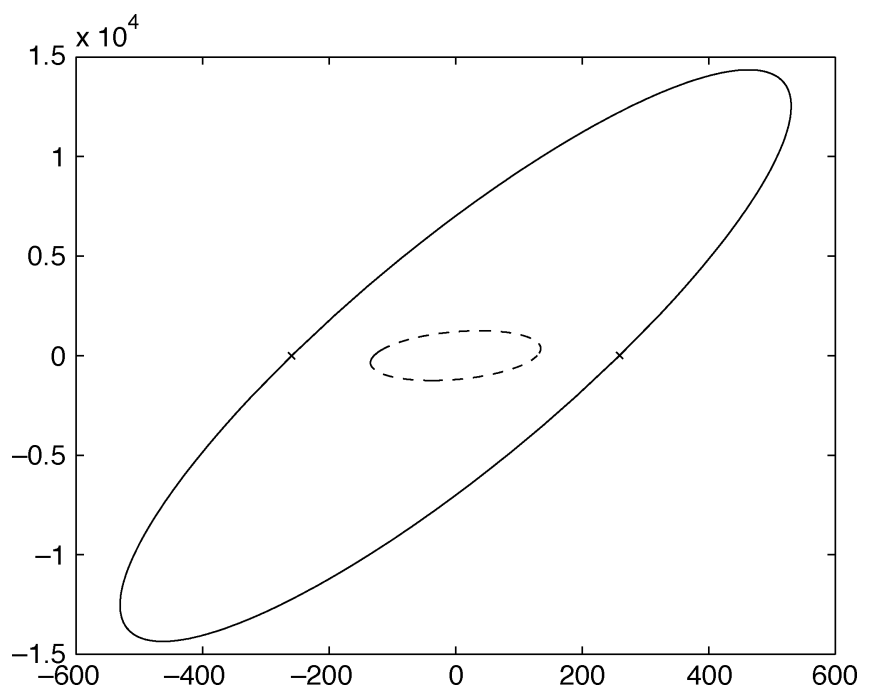

Fig. 3. Stability domains: Proposed approach (continuous). Approach presented in [12](dashed).

Example 3: Consider the following data of the benchmark example used in [11]:

$$
\begin{aligned}
A & =\left[\begin{array}{cc}
-0.1 & 0 \\
0 & -0.1
\end{array}\right] \quad B=\left[\begin{array}{ll}
1.5 & 4 \\
1.2 & 3
\end{array}\right] \quad C=\left[\begin{array}{ll}
1 & 0 \\
0 & 1
\end{array}\right] \\
A_{c} & =\left[\begin{array}{ll}
0 & 0 \\
0 & 0
\end{array}\right] \quad B_{c}=\left[\begin{array}{cc}
-1 & 0 \\
0 & -1
\end{array}\right] \\
C_{c} & =\left[\begin{array}{cc}
0.3333 & 0 \\
0 & -0.1
\end{array}\right] \quad D_{c}=\left[\begin{array}{cc}
-3.3333 & 0 \\
0 & 1
\end{array}\right] .
\end{aligned}
$$

The control bounds are given by $u_{0}=\left[\begin{array}{ll}1 & 1\end{array}\right]^{\prime}$.

In this case, the open-loop system is stable. Then, by applying the condition stated in Corollary 1 with a gain constraint $E_{c(i, j)}^{2} \leq 100$, one obtains $E_{c}=\left[\begin{array}{cc}9.6593 & 3.9260 \\ 8.2607 & -0.7235\end{array}\right]$ which stabilizes the system globally. It is worth to notice that the gain computed in [11] is only locally stabilizing.

Considering now the matrix $A=\left[\begin{array}{cc}0.1 & 0 \\ 0 & -0.1\end{array}\right]$ and keeping the other matrices as before, the closed-loop system without input saturation is still stable. As in [11], we take $\Xi_{0}=\left[\begin{array}{lllll}0.6 & 0.4 & 0 & 0\end{array}\right]^{\prime}$ Hence, from (19), and considering the gain limitation $E_{c(i, j)}^{2} \leq 10000$, one obtains

$$
\beta=36.6119 \quad E_{c}=\left[\begin{array}{rr}
96.1286 & -88.9092 \\
99.9998 & -100
\end{array}\right] .
$$

Without gain constraints the maximal achievable $\beta$ is 71.72 .

Taking the gain computed in [11] and applying the condition given in that paper in an analysis context it turns out that the maximal achievable $\beta^{1}$ is 36.2187 . In this case, the solutions of the two approaches are comparable. However, it should be noticed, that the solution given in [11] is obtained from a particular initialization of the iterative algorithm. In our case, the solution is direct and independent of any initialization.

\section{CONCLUDING REMARKS}

Given a dynamic output compensator that stabilizes the original system in the absence of saturation, we have proposed a method that allows to compute an antiwindup gain and an associated region of asymptotic stability for the closed-loop system with control saturation. In particular, we have focused on the problem of enlargement of the basin of attraction of the closed-loop system by using antiwindup gains. This problem has been indirectly addressed through a convex

\footnotetext{
${ }^{1}$ The value of 61.29 given in the note is apparently incorrect.
} 
optimization problem that allows to compute the antiwindup gain that leads to the maximization of an estimate of the basin of attraction.

The obtained stability conditions are based on a modified sector condition, that at our knowledge is new and original. The main advantage of the proposed approach with respect to the previous ones ([11] and [12]), is that the conditions are directly in an LMI form. Considering a criterion associated to the maximization of the stability region (estimate of the basin of attraction) is then possible to formulate the antiwindup synthesis problem directly as a convex optimization problem, avoiding the iterative schemes present in the previous approaches. Furthermore, it is shown that the results obtained with a classical sector condition are particular cases of the present one. On the other hand, compared with the approach that uses polytopic differential inclusions, the proposed condition is less complex. The effectiveness of the approach has been illustrated in a numerical example. It has also been shown that the proposed approach can be easily extended to treat systems presenting time-delays. On the other hand, following the ideas in [12], it should be possible to extend the approach to consider domains associated to Lure-Lyapunov functions.

\section{REFERENCES}

[1] H. A. Fertik and C. W. Ross, "Direct digital control algorithm with antiwindup feature," ISA Trans., vol. 6, pp. 317-328, 1967.

[2] K. J. Åström and L. Rundqwist, "Integrator windup and how to avoid it," in Proc. Amer. Control Conf., Pittsburgh, PA, 1989, pp. 1693-1698.

[3] C. Barbu, R. Reginatto, A. R. Teel, and L. Zaccarian, "Anti-windup for exponentially unstable linear systems with inputs limited in magnitude and rate," presented at the Amer. Control Conf., Chicago, IL, June 2000.

[4] M. V. Kothare and M. Morari, "Multiplier theory for stability analisys of anti-windup control systems," Automatica, vol. 35, pp. 917-928, 1999.

[5] A. R. Teel, "Anti-windup for exponentially unstable linear systems," Int. J. Robust Nonlinear Control, vol. 9, no. 10, pp. 701-716, 1999.

[6] C. Burgat and S. Tarbouriech, "Intelligent anti-windup for systems with input magnitude saturation," Int. J. Robust Nonlinear Control, vol. 8, pp. 1085-1100, 1998

[7] N. Kapoor, A. R. Teel, and P. Daoutidis, "An anti-windup design for linear systems with input saturation," Automatica, vol. 34, no. 5, pp. 559-574, 1998.

[8] A. R. Teel and N. Kapoor, "The $\mathcal{L}_{2}$ anti-windup problem: its definition and solution," presented at the Eur. Control Conf., Brussels, Belgium, July 1997.

[9] M. V. Kothare and M. Morari, "Stability analysis of anti-windup control scheme: A review and some generalizations," presented at the Eur. Control Conf., Brussels, Belgium, 1997.

[10] A. Miyamoto and G. Vinnicombe, "Robust control of plants with saturation nonlinearity based on coprime factor representation," in Proc. IEEE Conf. Decision and Control, Kobe, Japan, Dec. 1996, pp. 2838-2840.

[11] Y. Cao, Z. Lin, and D. G. Ward, "An antiwindup approach to enlarging domain of attraction for linear systems subject to actuator saturation," IEEE Trans. Autom. Control, vol. 47, pp. 140-145, Jan. 2002.

[12] J. M. Gomes da Silva, Jr., S. Tarbouriech, and R. Reginatto, "Analysis of regions of stability for linear systems with saturating inputs through an anti-windup scheme," presented at the IEEE Conf. Control Applications (CCA/CACSD'02), Glasgow, U.K., 2002.

[13] J. M. Gomes da Silva, Jr. and S. Tarbouriech, "Local stabilization of discrete-time linear systems with saturating controls: an LMI-based approach," IEEE Trans. Autom. Control, vol. 46, pp. 119-125, Jan. 2001.

[14] S. Boyd, L. El Ghaoui, E. Feron, and V. Balakrishnan, Linear Matrix Inequalities in System and Control Theory. Philadelphia, PA: SIAM, 1994.

[15] T. Hu and Z. Lin, "Exact characterization of invariant ellipsoid for single input linear systems subject to actuator saturation," IEEE Trans. Autom. Control, vol. 47, pp. 164-169, Jan. 2002.

[16] S. Tarbouriech, J. M. Gomes da Silva, Jr., and G. Garcia, "Delay-dependent anti-windup loops for enlarging the stability region of time-delay systems with saturating inputs," ASME J. Dyna. Syst., Measure., Control, vol. 125, no. 1, pp. 265-267, June 2003.

[17] — "A note on delay-dependent anti-windup loop for systems with saturating delayed-inputs," LAAS-CNRS, Toulouse, France, Tech. Rep. 04 178, 2004.

\section{Output Feedback Tracking: A Separation Principle Approach}

\author{
Manfredi Maggiore and Kevin M. Passino
}

\begin{abstract}
We study the practical and asymptotic tracking problems for nonlinear systems when only the output of the plant and the reference signal are available for feedback. We provide sufficient conditions and a control topology yielding practical tracking. In the special case when the reference signal is generated by an exosystem and there exists an internal model satisfying suitable observability properties, tracking becomes asymptotic.
\end{abstract}

Index Terms-Nonlinear control, nonlinear observer, output feedback, tracking.

\section{INTRODUCTION}

Consider the nonlinear system

$$
\begin{aligned}
& \dot{x}=f(x, u) \\
& y=h(x)
\end{aligned}
$$

where $x \in \mathbb{R}^{n}$ denotes the state of the system, $u \in \mathbb{R}^{m}$ is the control input, and $y \in \mathbb{R}^{m}$ is the measurable output. The vector field $f$ and the function $h$ are assumed to be sufficiently smooth. In this note, we address the following problem.

Problem 1 (Output Feedback Practical Tracking): Given the dynamical system (1), a sufficiently smooth reference trajectory ${ }^{1} r(t)=$ $\left[r_{1}(t), \ldots, r_{m}(t)\right]^{\top}$, and any real number $e_{0}>0$, find, if possible, an output feedback

$$
\begin{aligned}
\dot{x}_{c} & =f_{c}\left(x_{c}, y, r\right) \\
u & =h_{c}\left(x_{c}, y\right)
\end{aligned}
$$

with the property that for the closed-loop system (1) and (2), there exists a positive real number $T$ and a closed set $\mathcal{A}$ such that any integral curve $\left(x(t), x_{c}(t)\right)$ leaving from $\mathcal{A}$ is defined for all $t \geq 0$, bounded, and $\|y(t)-r(t)\| \leq e_{0}$ for all $t \geq T$.

If Problem 1 can be solved with $e_{0}=0$ and $T=\infty$, we say that (2) solves the output feedback asymptotic tracking problem. Additionally, if the projection $\left\{x \in \mathbb{R}^{n}:\left(x, x_{c}\right) \in \mathcal{A}\right\}$ can be made arbitrarily large by a suitable choice of the controller, we say that the solution to Problem 1 is semiglobal.

Problem 1 has been solved globally and asymptotically for systems in output feedback form [1], [2]. When the reference trajectory is generated by an exosystem, Problem 1 is included in the more general class of output regulation problems [3], where exosystem-generated disturbances and parametric uncertainties are allowed to affect the plant (our approach does not handle these). It has been shown, for

Manuscript received July 28, 2003; revised May 25, 2004 and September 10, 2004. Recommended by Associate Editor M. Reyhanoglu. This work was supported by the NASA Glenn Research Center under Grant NAG3-2084, and by the Natural Sciences and Engineering Research Council of Canada (NSERC). Part of this work was performed while the first author was at The Ohio State University, Columbus.

M. Maggiore is with the Department of Electrical and Computer Engineering, University of Toronto, Toronto, ON M5S 3G4, Canada (e-mail: maggiore@ control.utoronto.ca).

K. M. Passino is with the Department of Electrical Engineering, The Ohio State University, Columbus, OH 43210 USA (e-mail: k.passino@osu.edu).

Digital Object Identifier 10.1109/TAC.2004.841126

${ }^{1}$ We do not require to know the entire signal $r(t)$ in advance. 